\title{
Dynamical Test of Phase Transition Order
}

\author{
P. E. Cladis, ${ }^{(1)}$ Wim van Saarloos, ${ }^{(1)}$ David A. Huse, ${ }^{(1)}$ J. S. Patel, ${ }^{(2)}$ \\ J. W. Goodby, ${ }^{(1),(a)}$ and P. L. Finn ${ }^{(1)}$ \\ (1) AT\& T Bell Laboratories, Murray Hill, New Jersey 07974 \\ ${ }^{(2)}$ Bell Communications Research, Redbank, New Jersey 07791 \\ (Received 19 January 1988; revised manuscript received 11 October 1988)
}

\begin{abstract}
The dynamics of the interface between an ordered phase, characterized by an order parameter $\psi$ and a disordered phase where $\psi=0$, provide a promising, powerful tool to distinguish between continuous or second-order and weakly first-order phase transitions. We apply this idea to the nematic-smectic- $A$ transition and find that this transition is weakly first order even when the latent heat is too small to measure.

PACS numbers: $64.70 . \mathrm{Md}, 64.60 . \mathrm{Cn}, 68.35 . \mathrm{Rh}, 68.45 . \mathrm{Kg}$
\end{abstract}

Distinguishing an order-disorder transition that is weakly first order from one that is continuous can be difficult. The latent heat of the transition is small and thus difficult to measure. Also, the correlation length of fluctuations can grow to a very large, but finite, value as the transition is approached, further obscuring the difference between weakly first- and second-order (continuous) transitions. In this paper, we describe a novel method, especially useful for weakly first-order transitions, to experimentally infer the phase transition order from the existence and dynamical properties of an interface, or front, between two phases. We apply it to the smectic- $A$-nematic $(A-N)$ transition whose nature has been under discussion for over fifteen years. ${ }^{1-3}$

The order parameter $\psi$ of the $A$ phase is the amplitude of a one-dimensional density wave with wave vector parallel to the director $\mathbf{n}$. The $N$ phase is simply an anisotropic liquid with $\psi=0$ and the molecules on average aligned with n. In 1974, Halperin, Lubensky, and Ma $(\mathrm{HLM})^{2}$ predicted that the coupling between the fluctuations of $\psi$ and $\mathbf{n}$ results in a cubic term in the Landau-de Gennes free energy so that the $N-A$ transition is always first order. ${ }^{2}$

In spite of many sophisticated experiments, the nature of this transition has never been completely settled. ${ }^{3} \mathrm{Re}$ cently, Anisimov et al. ${ }^{4}$ showed that the scaling of increasingly smaller latent heats agrees with the existence of the HLM cubic term. However, some compounds have immeasurably small latent heats ${ }^{3-5}$ and so appear to be second order. By studying the dynamic behavior of an interface at this transition, we find that five samples in this latter class are actually first order. A longer paper $^{6}$ is required to show that our data are consistent with a HLM cubic term in the free energy.

In a Ginzburg-Landau analysis, the phase transition between the ordered $(A)$ phase with $\psi \neq 0$ and the disordered $(N)$ phase with $\psi=0$ is described by a free energy of the type 1

$$
F=\int_{V}[f(\psi)+\text { gradient terms }] d V,
$$

where $f(\psi)$ is a polynomial in $\psi$. HLM show that the coupling to director fluctuations results in a cubic term, $-\left.|\psi|\right|^{3}$, in $f(\psi)$, inevitably making this transition first order. ${ }^{2}$ Let us now summarize how interfacial properties depend on phase transition order in a mean-field picture.

For a second-order transition, $f(\psi)$ is such that above the transition temperature, $T_{c}, f$ has only one minimum at $\psi=0$. Furthermore, when $T>T_{c}, d f / d \psi \neq 0$ for any $\psi \neq 0$ providing a finite driving force for relaxation to the disordered state. This implies that if the system is brought into a state $\psi \neq 0$, e.g., by rapid heating, the order parameter relaxes homogeneously. Propagating interfaces cannot exist for $T>T_{c}$. Below $T_{c}, f$ has minima at $\psi \neq 0$ and a maximum at $\psi=0$. Although the disordered state is unstable the driving force vanishes, $d f / d \psi=0$, at $\psi=0$. As a result, a propagating front or interface can, in principle, be created for $T<T_{c}$. As has been shown ${ }^{7}$ and verified experimentally, ${ }^{8}$ the speed of such fronts varies as $|\epsilon|^{1 / 2}$, not linearly, with $\epsilon=(T$ $\left.-T_{c}\right) / T_{c}$.

Near a first-order phase transition, both the ordered and disordered phases are local minima of $f(\psi)$. Their relative stability depends on $T$. For $T>T_{c}$, the one at $\psi=0$ is stable while the one(s) at $\psi \neq 0$ is (are) metastable. When $T<T_{c}$, their stabilities are exchanged. Since both phases are locally stable at the transition, the free energy of an interface between the two phases is positive. There is a nucleation barrier near the transition so the system can be undercooled or superheated. Thus, at first-order transitions, interfaces occur and, depending on temperature, they propagate into either phase.

If the transition is first order, one gets from the timedependent Ginzburg-Landau equation, $\tau_{0} \partial \psi / \partial t=-\delta F /$ $\delta \psi$, for the speed $v$ of a steadily moving front between the disordered phase at $x \rightarrow-\infty$ and the ordered phase at $x \rightarrow+\infty$,

$$
v \tau_{0} \int_{-\infty}^{+\infty} d x\left(\frac{d \psi}{d x}\right)^{2}=\int_{-\infty}^{+\infty} d x \frac{d \psi}{d x} \frac{d f}{d \psi}=f_{s}-f_{n}=L \epsilon,
$$

where $L$ is the latent heat and $\tau_{0}$ is a microscopic relaxa- 
tion time. 9 Equation (2) relates the front speed, the front profile $d \psi / d x$, and the free-energy density difference between the two phases. It confirms that a transition to a lower free-energy state can occur by front propagation. Sufficiently near $T_{c}, f_{s}-f_{n}$ is linear in $\epsilon$, so from Eq. (2) $v$ goes linearly through zero at $T_{c}$. Of course, when the transition is weakly first order, one expects the linear regime in $v$ vs $\epsilon$ to be small. Next, using scaling arguments, we relate the front profile and latent heat to the coherence length $\xi_{c}$ at $T_{c}$ to obtain the slope of $v$ vs $\epsilon$.

If the HLM cubic term is absent, $f$ is of the form ${ }^{10}$ $f=\psi^{2}\left(\psi^{2}-\psi_{0}^{2}\right)^{2}$ near a Landau tricritical point (LTP) where the coefficient of the $\psi^{4}$ term is zero. The curvature at the $\psi=0$ minimum is given by $d^{2} f / d \psi^{2}=\psi_{0}^{4} \sim 1 /$ $\xi_{c}^{2}$ and the latent heat scales as $L \sim \psi_{0}^{2} \sim \xi_{c}^{-1}$. Substitution of these values into Eq. (2) gives $v \tau_{0} \psi_{0}^{2} / \xi_{c} \sim \epsilon / \xi_{c}$ or $v / \epsilon \sim \xi_{c} / \tau_{0}$. Thus, near the LTP, a linear relation is expected between the slope of $v(\epsilon)$ and $\xi_{c}$. The more first order the transition, the slower the front propagates. With a HLM cubic term, the LTP is not a tricritical point and the above linear scaling relation between $v / \epsilon$ and $\xi_{c}$ breaks down for small $\psi_{0}$ or $L .^{6}$

In summary, stable as well as moving interfaces occur near first-order transitions. The velocity is linear in $\epsilon$ for $\epsilon \rightarrow 0$. For second-order phase transitions, interfaces can be created under carefully controlled experimental conditions only on one side of the transition, and then they propagate with a speed proportional to $\epsilon^{1 / 2}$.

We now apply these ideas to fronts propagating under isothermal conditions in the two-component systems $8 \mathrm{CB}-10 \mathrm{CB}$ and $9 \mathrm{CB}-10 \mathrm{CB} .{ }^{11}$ These mixtures are of particular interest because the greater the concentration of $10 \mathrm{CB}$ the more first order the $N-A$ transition. The best available $\mathrm{x}$-ray ${ }^{12}$ measurements show this transition to be second order in $8 \mathrm{CB}$ and find a tricritical point at $9.7 \%$ by weight $10 \mathrm{CB}$ in $9 \mathrm{CB}$. On the other hand, the best available calorimetry measurements find the 9CB$10 \mathrm{CB}$ system to be always first order and a tricritical point at $32 \% 10 \mathrm{CB}$ in $8 \mathrm{CB}$ in the $8 \mathrm{CB}-10 \mathrm{CB}$ system. ${ }^{5}$ In our experiments on these two systems, however, we always observe interfaces (see inset of Fig. 1) whose properties are consistent with the $N-A$ transition being weakly first order. ${ }^{11}$

The experiment was to start from a uniform state above (or below) but within $0.02^{\circ} \mathrm{C}$ of $T_{c}$, rapidly change the temperature to $T$, just below (or above) $T_{c}$, wait typically a few seconds for the front to appear, and record the front passage with a video monitor. From a frame-by-frame analysis, the speed $v(\epsilon)$ is found, with a resolution of $0.1 \mathrm{sec}$, from the time the interface takes to travel about $1 \mathrm{~mm}$. A plot of $v$ as a function of temperature from the $8 \mathrm{CB}-10 \mathrm{CB}$ study is shown in Fig. 2. For the $35 \mathrm{~mol} \%$ mixture, the front velocity clearly goes linearly through zero as expected for "weakly" firstorder transitions with just barely measurable latent

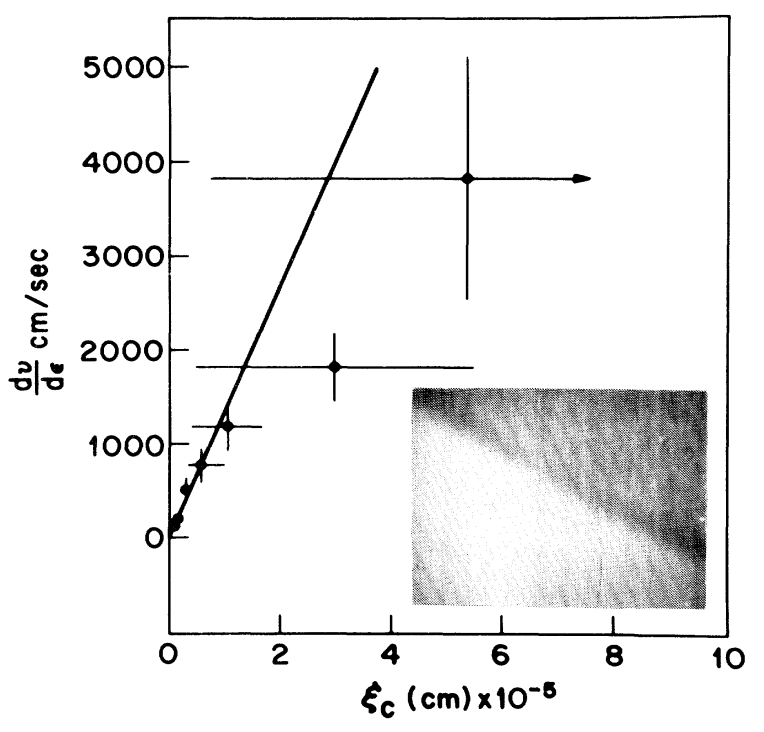

FIG. 1. Inset: Photograph of video monitor screen showing interface between the nematic phase (the darker region) and the smectic- $A$ phase. The direction of orientational order is parallel to the vertical axis. The sample is $9 \mathrm{CB}$ and the field of view $\sim 1 \mathrm{~mm}^{2}$. $d v / d \epsilon$ is plotted as a function of $\xi_{c}$. From left to right, the points represent the following concentrations: 28.2 , $22.4,15.6,9.7,5.8$, and 0.0 weight $\% 10 \mathrm{CB}$ in $9 \mathrm{CB}$ and $8 \mathrm{CB}$. The lower limit in the uncertainty of $\xi_{c}$ is the last data point measured by $x$ rays (Ref. 12) and the upper limit is simply chosen to be symmetric about $\xi_{c}$ estimated from latent heat data (Ref. 5). The error in speed is shown as $\pm 20 \%$ for the $9 \mathrm{CB}-10 \mathrm{CB}$ mixtures and $\pm 50 \%$ in $8 \mathrm{CB}$.

heats. Although the uncertainty in temperature is the same for $8 \mathrm{CB}$ as for the mixture, its steepness precludes as precise a determination of the slope as for the mixture. Nevertheless, these data are also consistent with a linear dependence of $v$ on $\epsilon$. Our data for the $9 \%$ and $17 \%$ mixtures, both with latent heats too small to measure, ${ }^{5}$ fall between these two extremes. These data are shown as an inset to Fig. 2.

Temperature gradients, in principle, can create apparent interfaces at second-order transitions. The following observations provide strong arguments against this possibility in our experiments. (i) Small $(2 \times 2.5$ $\times 0.7 \mathrm{~mm}^{3}$ ) platinum resistance thermometers, thermally sunk to the cells, measured the temperature accurately to $0.01^{\circ} \mathrm{C}$. The data presented here were all taken at constant temperature. (ii) Cells made of two glass plates 1 mm thick or two sapphire plates $0.5 \mathrm{~mm}$ thick with the liquid crystal in the $13-\mu \mathrm{m}$ gap between the plates were used. Although the thermal diffusivity of sapphire is 26 times larger than glass, within the experimental accuracy of $20 \%$, the measured velocities are the same in both types of cells. Thus, the interface motion is not driven by thermal relaxation of the cell and not governed by 


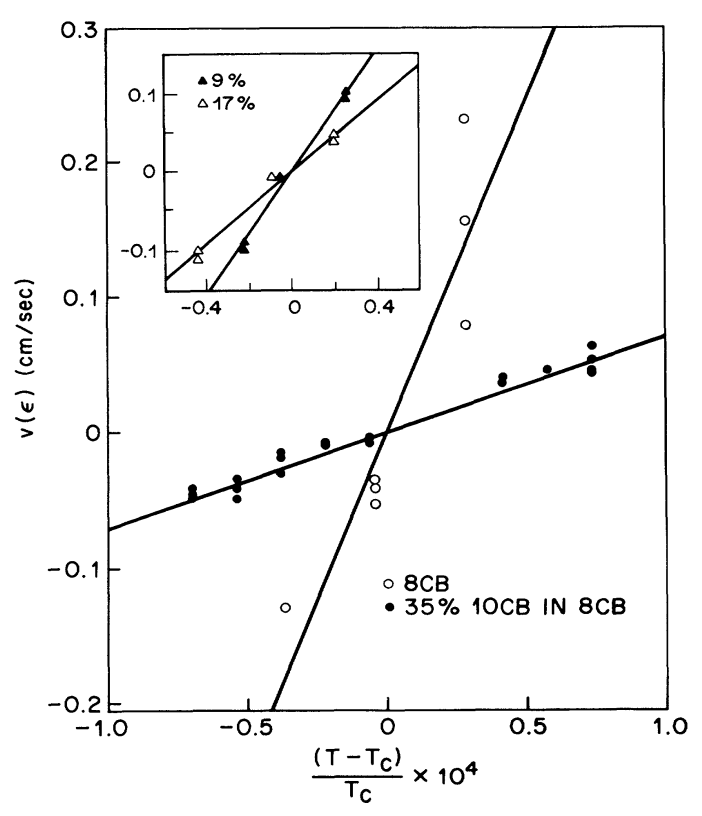

FIG. 2. Speed as a function of temperature for $8 \mathrm{CB}$ and 35 mol\% $10 \mathrm{CB}$ in $8 \mathrm{CB}$. Inset: Similar data for 9 and $17 \mathrm{~mol} \%$ $10 C B$ in $8 \mathrm{CB}$ mixtures. Observing an interface propagate on both heating and cooling is qualitatively the signature of a first-order transition.

heat release in the interfacial region. ${ }^{13}$ (iii) Our data do not show a detectable asymmetry for cooling and heating. ${ }^{14} \mathrm{~A}$ quantitative discussion of the expected asymmetry of a gradient-induced interface at a second-order transition will be given elsewhere. ${ }^{6}$ (iv) Our field of view is always at a fixed position with respect to the heaters. With a given part of the sample cell in the field of view, the interface propagates in a fixed direction with respect to the sample, presumably determined by a nucleation site. When a different part of the cell is brought into the field of view, the propagation direction is different with respect to the heaters. It is, therefore, unlikely that systematic temperature gradients play a role. (v) In a narrow range around $T_{c}$, we observed static interfaces pinned at certain spots, presumably imperfections, of the cell. Pinning is associated with a surface tension between the two phases, a feature of first-order transitions.

It is known ${ }^{1}$ that bend or twist distortions can inhibit the formation of the $A$ phase and drive the transition first order. Indeed, we found it important to have cells of uniform thickness, $13 \pm 2 \mu \mathrm{m},{ }^{15}$ with the director oriented in the plane of the substrate ${ }^{16}$ and the alignment on both surfaces parallel to within $1^{\circ}$. The director was set parallel to the polarizer and the analyzer slightly offset from the crossed position. Apparently the observed contrast results from the strong decrease in Rayleigh scattering in the $A$ phase, although it is surprising how strong the contrast is when the transition is very weakly first order (e.g., pure 8CB).

From data similar to those shown in Fig. 2, we determined the slope $d v / d \epsilon$ with a linear least-squares fit. The $\xi_{c}$ are not known for the 8CB-10CB mixtures, so we only analyze the $9 \mathrm{CB}-10 \mathrm{CB}$ mixtures to check the scaling relation $v / \epsilon \sim \xi_{c}$. Although the coherence length parallel $\left(\xi_{\|}\right)$to $\mathbf{n}$ is about 10 times larger than perpendicular $\left(\xi_{\perp}\right)$ to $\mathbf{n}$, we surprisingly did not detect a corresponding systematic anisotropy in the propagating speed. Since there was no direction dependence of $v$, we took $\xi_{c}$ to be the average that enters in hyperscaling, ${ }^{17}$ $\xi_{c}=\left[\xi_{\|}\left(T_{c}\right) \xi_{\perp}^{2}\left(T_{c}\right)\right] .{ }^{1 / 3}$ Using the available latent heat ${ }^{5}$ and $\mathrm{x}$-ray ${ }^{12}$ data, we verified the relationship $L \sim \xi_{c}{ }^{-1}$ over the range 14\%-28\% 10CB in 9CB (a factor of 3 in $\xi_{c}$ ) and used it to estimate $\xi_{c}$ for concentrations below $14 \%$ where measured values of $\xi_{c}$ are unavailable. An upper limit for the latent heat is used to estimate $\xi_{c}$ for $8 \mathrm{CB} .{ }^{5}$ The lower limits on the error bars on $\xi_{c}$ in Fig. 1 represent the largest $\xi_{c}$ seen in the x-ray data ${ }^{12}$ for each sample. Figure 1 shows $d v / d \epsilon$ vs $\xi_{c}$ so obtained for the concentrations studied by Ocko, Birgenaue, and Litster. ${ }^{12}$

In Fig. 1, each data point represents a different mixture corresponding to the composition range $0 \%-28.2 \%$ $10 C B$ in $9 C B$ and pure $8 C B$. Using only points where the uncertainty in $\xi_{c}$ is insignificant, the straight line describing the data is $d v / d \epsilon=\xi_{c} / \tau_{0}$ with microscopic time $\tau_{0}=7.5 \times 10^{-9} \mathrm{sec}$. Taking a typical diffusion constant for the $N$ and $A$ phases, $D=4 \times 10^{-7} \mathrm{~cm}^{2} / \mathrm{sec}$, at similar temperatures, ${ }^{18}$ in time $\tau_{0}$ a molecule diffuses $\sim 6 \AA$, adequate to relax the smectic order parameter. A cubic HLM term in the free energy would result in a crossover at large $\xi_{c}$ to a slower increase of $d v / d \epsilon$ with $\xi_{c}{ }^{6}$ The latent heat data suggest that such a crossover is operative for pure $9 \mathrm{CB}$ and $8 \mathrm{CB} .^{6}$

The magnitude of $d v / d \epsilon \sim 10 \mathrm{~m} / \mathrm{sec}$ in Fig. 1 shows that $N-A$ interfaces grow nearly as easily as solid-liquid interfaces in simple atomic systems. Computer simulations on Lennard-Jones systems and experimental data on $\mathrm{Si}$ are consistent with a slope of the same magnitude. Clearly, the weakness of the first-order transition contributes to the rather fast growth of these liquid-crystal interfaces.

By combining the fact that it is unusual, requiring considerable experimental skill, ${ }^{8}$ to observe a moving front at second-order transitions with the fact that the dynamical signature of first-order transitions is qualitatively different from second-order transitions, we have proposed a novel experimental method to probe phase transition order. We find experimentally that the dynamic signature of the nematic-smectic- $A$ phase transition in many compounds, ${ }^{11}$ even some with latent heats too small to measure, is consistent with a first-order transition. Making contact with the coherence length at $T_{c},{ }^{5,12}$ we found in a regime (small enough $\xi_{c}$, large enough latent heat) where the HLM cubic term is still 
small, that $d v(\epsilon) / d \epsilon=\xi_{c} / \tau_{0}$ with $\tau_{0}=7.5 \times 10^{-9}$ sec. The more first order the transition, the slower the front propagates. In a longer version of this paper, ${ }^{6}$ it will be shown that data for smaller latent heats are consistent with a crossover to the scaling form associated with a HLM cubic term. ${ }^{2}$ Our dynamic measurements also pose new questions that deserve further study: (i) Why is the observed contrast so large? (ii) How does director relaxation influence interface dynamics? (iii) Why is a systematic dependence of the interface velocity on the direction of propagation not observed?

It is a pleasure to thank M. A. Anisimov, Ph. Nozieres, and P. Ukleja for stimulating discussions and Christopher Arzt for help in sample preparation.

\footnotetext{
(a) Present address: School of Chemistry, The University of Hull, Hull HU6 7RX, England.

${ }^{1}$ P. G. de Gennes, Solid State Commun. 10, 753 (1972).

${ }^{2}$ B. I. Halperin, T. C. Lubensky, and S. K. Ma, Phys. Rev. Lett. 32, 292 (1974); B. I. Halperin and T. C. Lubensky, Solid State Commun. 14, 997 (1974).

${ }^{3}$ For reviews, see D. L. Johnson, J. Chim. Phys. Phys. Chim. Biol. 8045 (1983); J. D. Litster, Philos. Trans. Roy. Soc. London A 309, 145 (1983); T. C. Lubensky, J. Chim. Phys. Phys. Chim. Biol. 80, 6 (1983); Peter Pershan, Structure of Liquid Crystal Phases (World Scientific, Singapore, 1988).

${ }^{4}$ M. A. Anisimov, V. P. Voronov, E. E. Gorodetskii, V. E. Podnek, and F. Kholmudorov, Pis'ma Zh. Eksp. Teor. Fiz. 45, 336 (1987) [JETP Lett. 45, 425 (1987)].

${ }^{5}$ J. Thoen, H. Marynissen, and W. van Dael, Phys. Rev. Lett. 52, 204 (1984); H. Marynissen, J. Thoen, and W. van Dael, Mol. Cryst. Liq. Cryst. 124, 195 (1985); J. Thoen, H. Marynissen, and W. van Dael, Phys. Rev. A 26, 2886 (1982).
}

${ }^{6}$ M. A. Anisimov, P. E. Cladis, E. E. Gorodetskii, D. A. Huse, V. G. Taratuta, W. van Saarloos, and V. P. Voronov (to be published).

${ }^{7}$ See, e.g., W. van Saarloos, Phys. Rev. A 37, 211 (1988), and references therein.

${ }^{8}$ J. Fineberg and V. Steinberg, Phys. Rev. Lett. 58, 1332 (1987).

${ }^{9}$ Equation (2) equates the total dissipation $\int d x\left(\tau_{0} / v\right)(\partial \psi /$ $\partial x)^{2}$ to the free-energy gain. To obtain Eq. (2), note that the time-dependent Ginzburg-Landau equation with $\psi=\psi(x=u$ $-v t$ ) yields $v d \psi / d x=\delta F / \delta \psi$. A similar argument is often used to calculate the speed of defects [see, e.g., P. E. Cladis, W. van Saarloos, P. L. Finn, and A. R. Kortan, Phys. Rev. Lett. 58, 222 (1987)] or kinks [see, e.g., M. Buttiker and H. Thomas, Phys. Rev. A 37, 235 (1988), and references therein].

${ }^{10}$ R. B. Griffiths, J. Chem. Phys. 60, 195 (1974).

${ }^{11} n$-CB is $n$-alkyl cyanobiphenyl. Here we report data taken on the following: 9CB; the $9 \mathrm{CB}-10 \mathrm{CB}$ mixtures, $4.7,5,5.8$, $8.9,9,9.7,14.1,14.2,15.6,20.1,22.4$, and 28.1 weight $\% 10 \mathrm{CB}$ in $9 \mathrm{CB} ; 8 \mathrm{CB}$; the $8 \mathrm{CB}-10 \mathrm{CB}$ mixtures, $10,18,38$, and 50 weight $\% 10 \mathrm{CB}$ in $8 \mathrm{CB}$. Moreover, $8 \mathrm{OCB}, 1.5 \% 6 \mathrm{OCB}$ in $8 \mathrm{OCB}, 15 \%, 20 \%$, and $32 \% 8 \mathrm{OCB}$ in DB7OCN, 40.8 and $1.5 \% 4 \mathrm{O} .8$ in $8 \mathrm{OCB}$ showed qualitatively similar results.

${ }^{12}$ B. M. Ocko, R. J. Birgeneau, and J. D. Litster, Z. Phys. B 62, 487 (1986).

${ }^{13}$ Note that according to the measurements in Thoen, Marynissen, and van Dael, Ref. 5, the specific heat of $8 \mathrm{CB}$ is sharply peaked within an interval of $0.02^{\circ} \mathrm{C}$, so that even an (temperature gradient induced) effective interface at a second-order transition would behave thermally like a sharp interface with a latent heat if the temperature difference across the interface is larger than $0.02^{\circ} \mathrm{C}$. Temperature gradients of this magnitude certainly do not occur in our cell.

${ }^{14}$ Zone refining effects in mixtures would lead to asymmetric (about $T_{c}$ ) propagation speeds, also inconsistent with our data. Indeed, as pointed out by J. Bechhoeffer, P. Oswald, A. Libchaber, and C. Germain [Phys. Rev. A 37, 1691 (1988)] these effects are formally absent at second-order transitions and small at weakly first-order transitions.

${ }^{15}$ The cell thickness in the 8 OCB studies ranged from 2 to 75 $\mu \mathrm{m}$; however, no systematic dependence of the front speed on thickness was observed. $13 \mu \mathrm{m}$ was chosen for these studies because it is easy to fabricate, thin enough to avoid vertical temperature gradients, and a convenient thickness for observations in the optical microscope.

${ }^{16}$ J. S. Patel, T. M. Leslie, and J. W. Goodby, Ferroelectrics 59, 137 (1984).

${ }^{17}$ This is the anisotropic generalization of the hyperscaling relation of P. C. Hohenberg, A. Aharony, B. I. Halperin, and E. D. Siggia, Phys. Rev. 13, 2986 (1976), that often applies to the $N-A$ transition (Ref. 3 ).

${ }^{18}$ M. Hara, H. Takezoe, and A. Fukuda, Jpn. J. Appl. Phys. 25, 1756 (1986); G. J. Kruger, Phys. Rep. 82, 249 (1982). 


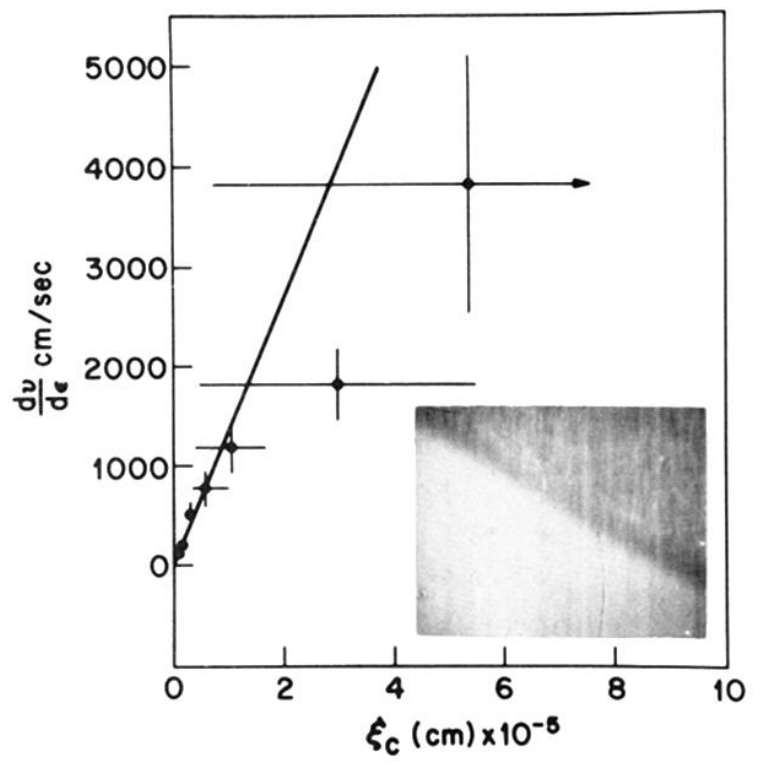

FIG. 1. Inset: Photograph of video monitor screen showing interface between the nematic phase (the darker region) and the smectic- $A$ phase. The direction of orientational order is parallel to the vertical axis. The sample is $9 \mathrm{CB}$ and the field of view $\sim 1 \mathrm{~mm}^{2}$. $d v / d \epsilon$ is plotted as a function of $\xi_{c}$. From left to right, the points represent the following concentrations: 28.2 , $22.4,15.6,9.7,5.8$, and 0.0 weight $\% 10 \mathrm{CB}$ in $9 \mathrm{CB}$ and $8 \mathrm{CB}$. The lower limit in the uncertainty of $\xi_{c}$ is the last data point measured by $x$ rays (Ref. 12) and the upper limit is simply chosen to be symmetric about $\xi_{c}$ estimated from latent heat data (Ref. 5). The error in speed is shown as $\pm 20 \%$ for the $9 \mathrm{CB}-10 \mathrm{CB}$ mixtures and $\pm 50 \%$ in $8 \mathrm{CB}$. 